\title{
Stacking effect and Coulomb correlation in layered charge density wave phase of $1 T-\mathrm{NbS}_{2}$
}

Wei Wang, ${ }^{1,2}$ Chen Si ${ }^{2}$ Wen Lei, ${ }^{1,3}$ Feng Xiao, ${ }^{1}$ Yunhui Liu, ${ }^{1}$ Carmine Autieri, ${ }^{4}$ and Xing Ming $1,5^{*}$

1. College of Science, Guilin University of Technology, Guilin 541004, PR China

2. School of Materials Science and Engineering, Beihang University, Beijing 100191, PR China

3. Key Laboratory of Artificial Micro- and Nano-Structures of Ministry of Education and School of Physics and Technology, Wuhan University, Wuhan 430072, PR China

4. International Research Centre MagTop, Institute of Physics, Polish Academy of Sciences, Aleja Lotników 32/46, PL-02668 Warsaw, Poland

5. MOE Key Laboratory of New Processing Technology for Nonferrous Metal and Materials, Guangxi Key Laboratory of Optic and Electronic Materials and Devices, Guilin University of Technology, Guilin 541004, PR China

\begin{abstract}
Two-dimensional (2D) layered materials have attracted tremendous interest from the perspective of basic physics and technological applications in the last decade. Especially, the artificially assembled van der Waals (vdW) heterostructures and twisted 2D materials bring out fascinating properties, and render promising applications possible by engineering the stacking order. Here, based on first-principles calculations, we explored the interplay between stacking effect and electron-electron correlation in the layered vdW material of bulk $1 T-\mathrm{NbS}_{2}$ with a $2 \mathrm{D}$ charge density wave (CDW) order. Without considering the Coulomb correlation, two energetically favorable out-of-plane stacking configurations are identified: one is a metallic phase with a single-layer stacking pattern, another is a band insulator with a paired-bilayer stacking configuration. Even though the Coulomb correlation is taken into account, the two energetic favorable stacking orders are still far more stable than other stacking orders. Furthermore, increasing the Coulomb interaction, the paired-bilayer stacking configuration transforms from nonmagnetic band insulator to antiferromagnetic insulator, while the single-layer stacking undergoes a Slater-Mott metal-insulator transition, which indicates the non-negligible role of electron-electron correlation interactions. In addition, the electronic structure and magnetic ground state change drastically among different stacking configurations, providing a platform to tune the electronic structures and interlayer magnetic interactions by altering the stacking order. In contrast to the widely accepted scenario of Mott localization as the driving force behind the gap formation in the CDW phase of layered transition metal dichalcogenides, our results not only highlight the crucial role of stacking order in the electronic structures of $1 T-\mathrm{NbS}_{2}$, but also shed fresh light on the distinct effects of Coulomb interaction in different stacking arrangements.
\end{abstract}

\footnotetext{
Email: mingxing@glut.edu.cn (Xing Ming)
} 


\section{INTRODUCTION}

Two-dimensional (2D) layered materials exhibit diverse properties owing to the delicate interaction between the layers held together by weak van der Waals (vdW) forces, which attract considerable attention as potential materials for promising applications.[1-3] Furthermore, abundant physical properties of the $\mathrm{vdW}$ layered materials can be modulated by the interlayer interactions through intercalation, pressure, strain, twist or stacking order. For example, the rotational degrees of freedom between layers can be controlled over to create new phenomena in known materials. Manipulating the twist angle between two 2D monolayers in vdW bilayer heterostructures can tune the band structures and electrical properties to realize twistronics devices.[4,5] In particular, the discovery of unconventional superconductivity, correlated insulating behavior, ferromagnetism and topological phase in the magic-angle twisted bilayer graphene have sparked researchers' unprecedented enthusiasm.[6-11]

On the other hand, lateral translation or sliding of one layer relative to another layer can modify the stacking order and stacking symmetry of the vdW layered materials, which gives rise to rich phase diagrams and novel properties, such as the stacking-engineered ferroelectricity in bilayer BN [12,13], stacking-tunable magnetism in bilayer $\mathrm{CrI}_{3}$ and $\mathrm{CrBr}_{3}$ [14,15], and stacking-driven metal-insulator transitions in layered $1 T-\mathrm{TaS}_{2}[16-19] .1 T-\mathrm{TaS}_{2}$ is an archetypal charge density wave (CDW) material that has been in the spotlight for many years, which transforms into a commensurate $\mathrm{CDW}$ (CCDW) phase characterized by $\sqrt{13} \times \sqrt{13}$ star-of-David (SD) clusters (schematically shown in Figure 1) upon cooling to $200 \mathrm{~K}$. Recently, rekindled interest in $1 T-\mathrm{TaS}_{2}$ stems from the extensive debate on the origin of the insulating nature in the CCDW phase. According to traditional wisdom, there is one unpaired electron forming a half-filled band in the CCDW phase, therefore, the insulating ground state is driven by the Mott-Hubbard mechanism.[20] However, first-principles density functional theory (DFT) calculations combined with transport measurements, angle-resolved photoemission spectroscopy (ARPES), low-energy electron diffraction experiments and atomic resolution scanning transmission electron microscopy (STEM) give robust evidence that the insulating gap of the CCDW phase is primarily governed by the out-of-plane stacking arrangements of the SD clusters rather than the electron-electron correlation.[16-19,21-29] These theoretical and experimental results highlight the critical role played by the stacking order of the SD clusters intertwining with 
orbital order in determining the electronic structure of the CCDW phase.
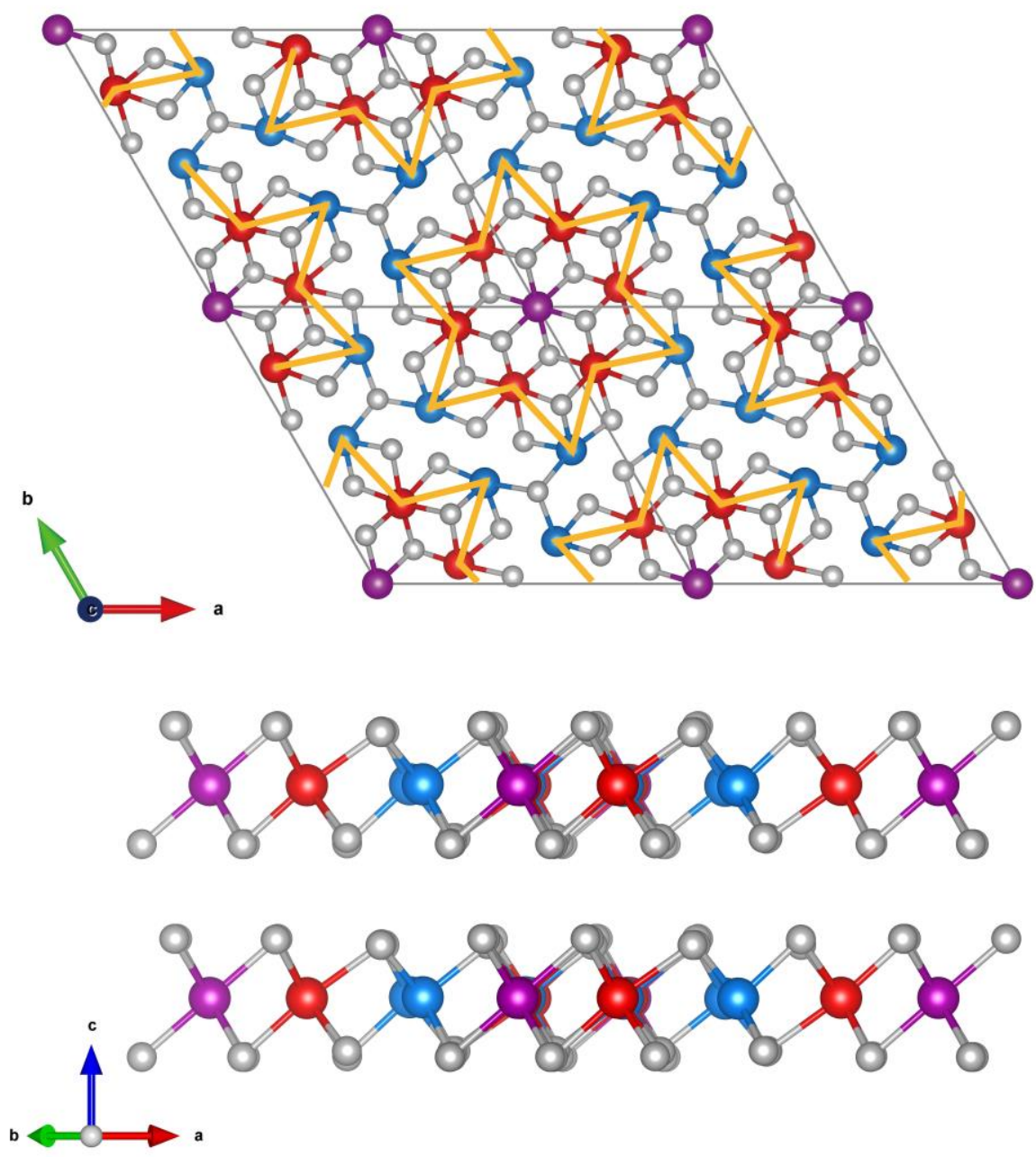

Figure 1 Top and side views of the CCDW phase of $1 T$ polytype $\mathrm{TaS}_{2}$ or $\mathrm{NbS}_{2}$ characterized by the $\sqrt{13} \times \sqrt{13}$ SD clusters (schematically drawn in yellow line). The big balls denote the Ta or $\mathrm{Nb}$ atoms (the purple, red and blue colors indicate three different structural symmetric sites), and the small balls (gray color) represent the $\mathrm{S}$ atoms.

Recently, we and Tresca et al. theoretically predicted that $1 T-\mathrm{NbS}_{2}$ belonging to $\mathrm{CDW}$ materials, which is isostructural and isovalent to $1 T-\mathrm{TaS}_{2}$ and also exhibits the typical $\sqrt{13} \times \sqrt{13}$ SD structural reconstructions (Figure 1).[30,31] In the bulk phase, the unit cell of $1 T$ polytype $\mathrm{NbS}_{2}$ consists of corner-sharing monolayer $\mathrm{NbS}_{6}$ octahedra, which differs from the bilayer and trilayer stacking arrangements of the corner-sharing trigonal prismatic coordination $\mathrm{NbS}_{6}$ layers in $2 H$ and $3 R$ polytypes $\mathrm{NbS}_{2}$.[32-34] The distinct stacking arrangements of the coordination polyhedra lead to dramatically different electronic structures ranging from insulator to metal to superconductor for these three polytypes of layered $\mathrm{NbS}_{2}$.[30-36] The monolayer $1 T-\mathrm{NbS}_{2}$ exhibits a ferromagnetic (FM) spin $1 / 2$ insulating state,[31] while an interlayer antiferromagnetic (AFM) 
ordering may dominate the interlayer coupling in the bulk phase [30]. Diffuse X-ray scattering measurements detect traces of $\mathrm{CDW}$ in $2 \mathrm{H}-\mathrm{NbS}_{2}$, which is also attributed to the local $1 T$ polytype environment at the interface of the rotational stacking faults between $2 H$ domains.[34] Martino et al. propose that the magnetic $\mathrm{CDW}$ state of the $1 T-\mathrm{NbS}_{2}$ layers can intercalate the host of $2 \mathrm{H}$ stacking $\mathrm{NbS}_{2}$ and form a $2 H / 1 T-\mathrm{NbS}_{2}$ heterostructure, and result in an unexpected unidirectional Kondo scattering in metallic $2 H-\mathrm{NbS}_{2}$.[36] The stacking order is expected to create lateral shifts of the $\mathrm{CDW}$ clusters between adjacent layers and provide an effective route to manipulate the electronic phases of the layered $1 T-\mathrm{NbS}_{2}$. Furthermore, being the same as the CCDW phase of $1 T-\mathrm{TaS}_{2}$, the unit cell of $1 T-\mathrm{NbS}_{2}$ is reconstructed into a triangular lattice with prototypical $\sqrt{13} \times \sqrt{13}$ SD clusters, and there is a single unpaired electron with $S=1 / 2$ spin moment localizing in the centers of each SD cluster.[30,31] Therefore, exotic quantum phases, such as quantum spin liquid, are expected to emerge in these triangular-lattice materials.[37-42]

The motivation of the present paper is to further examine the out-of-plane stacking order of the SD clusters in the CCDW phase by first-principles DFT calculations, and uncover the role played by the stacking order and electron-electron correlation in the stability and electronic phase of $1 T-\mathrm{NbS}_{2}$. We not only unveil the tunable electronic structures derived from the distinct stacking order of the SD clusters, but also reveal a significant effect of the Coulomb correlation in manipulating the electronic properties of the CCDW phase $1 T-\mathrm{NbS}_{2}$. We hope our theoretical results will stimulate further experimental and theoretical studies on the electronic properties of the CCDW phase to better understand the intricate interplay between the stacking order and electron correlation in $1 T-\mathrm{NbS}_{2}$. Furthermore, although we examine the case of the $1 T-\mathrm{NbS}_{2}$, similar effects could be present in other members of the same material class.

\section{STRUCTURE MODELS AND COMPUTATIONAL DETAILS}

The routinely stacked CCDW phase of $1 T-\mathrm{NbS}_{2}$ can be described by the space group of $\mathrm{P} \overline{3}$. Each $\mathrm{Nb}$-atom is bonded to six $\mathrm{S}$-atoms forming distorted octahedral coordination, and the adjacent layers are bonded together by weak vdW forces (the schematic model is presented in Figure 1). The building block of the CCDW phase is the SD cluster as shown in Figure 2(a). Due to the threefold in-plane rotational symmetry of the CCDW phase, five kinds of Nb-atoms can be defined, where the central $\mathrm{Nb}$-atom is labeled as 0 and the surrounding $\mathrm{Nb}$-atoms are labeled as 1 
to 4 in each SD cluster, respectively. Taking the threefold in-plane symmetry into account, the out-of-plane alignments of the SD clusters in adjacent planes are described by five types of stacking interfaces $t_{\mathrm{i}}(\mathrm{i}=0,1,2,3,4)$ [illustrated in Figures 2(b)-(f)], which lead to five types of out-of-plane 3D stacking order $T_{\mathrm{i}}(\mathrm{i}=0,1,2,3,4)$. The routinely stacking configuration $T_{0}$ corresponds to the on-top stacked monolayer with the stacking interface $t_{0}$ [Figures 2(b)], whereas other single-layer stacking configuration $T_{\mathrm{i}}$ can be viewed as a lateral shift [the dashed line depicted in Figures 2(c)-(f)] of every single layer with respect to the adjacent layer with the corresponding stacking interface $t_{\mathrm{i}}$. Accordingly, the central $\mathrm{Nb}$ atoms of the SD in the upper layer are located above the $\mathrm{Nb}$ atom in one of the tips ( $t_{1}$ and $t_{4}$ interfaces) or the inner circle hexagon ( $t_{2}$ and $t_{3}$ interfaces) of the SD of the lower layer. In addition, we also considered paired-bilayer stacking configurations with a lateral sliding of every two layers due to the possibility of the Peierls dimerization for a chain of half-filled bands. These four types of stacking order $T_{0 \mathrm{i}}(\mathrm{i}=1,2$, $3,4)$ can be viewed as on-top stacked bilayers with the stacking interface $t_{0}$, which by themselves are stacked by a stacking interface $t_{\mathrm{i}}(\mathrm{i}=1,2,3,4)$ in the out-of-plane direction.[16,17,21,26] All these stacking orders are illustrated detailly in Figure 3.

(a)

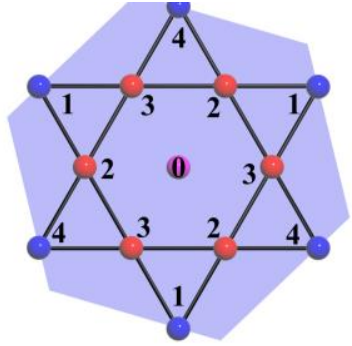

(d)

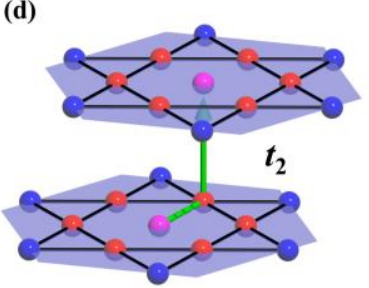

(b)

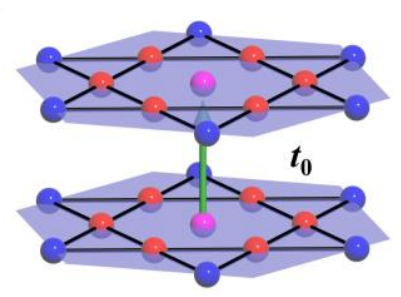

(e)

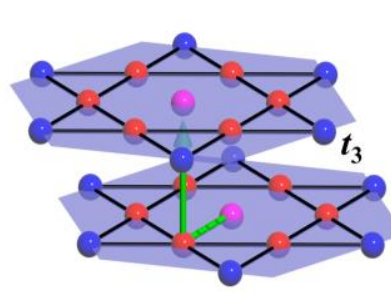

(c)

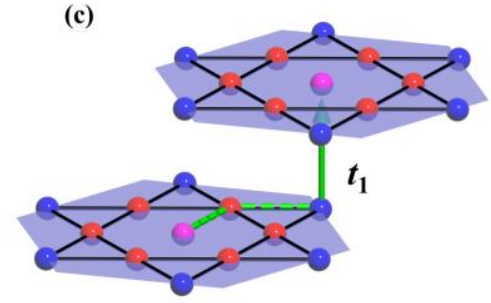

(f)

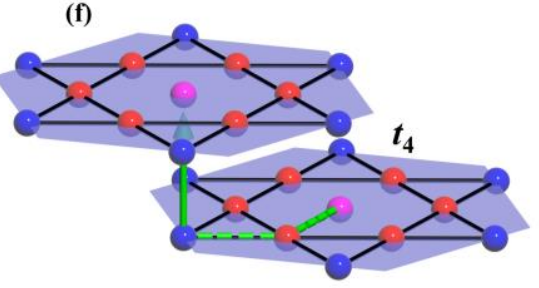

Figure 2 Schematic illustration of the $\sqrt{13} \times \sqrt{13}$ SD cluster in the CCDW phase of $1 T$ polytype $\mathrm{NbS}_{2}$ and different out-of-plane stacking order (the $\mathrm{S}$ atoms are not shown). (a) In-plane SD cluster and classification of the Nb-atoms. (b)-(f) are five kinds of stacking interfaces $\left(t_{0}-t_{4}\right)$ between two adjacent layers. Solid green arrows illustrate the $\mathrm{Nb}$-atom in the first layer facing to the central $\mathrm{Nb}$-atom in the adjacent layer, and dashed green lines denote the in-plane lateral shift of the SD clusters with respect to the counterparts in the adjacent layer. 

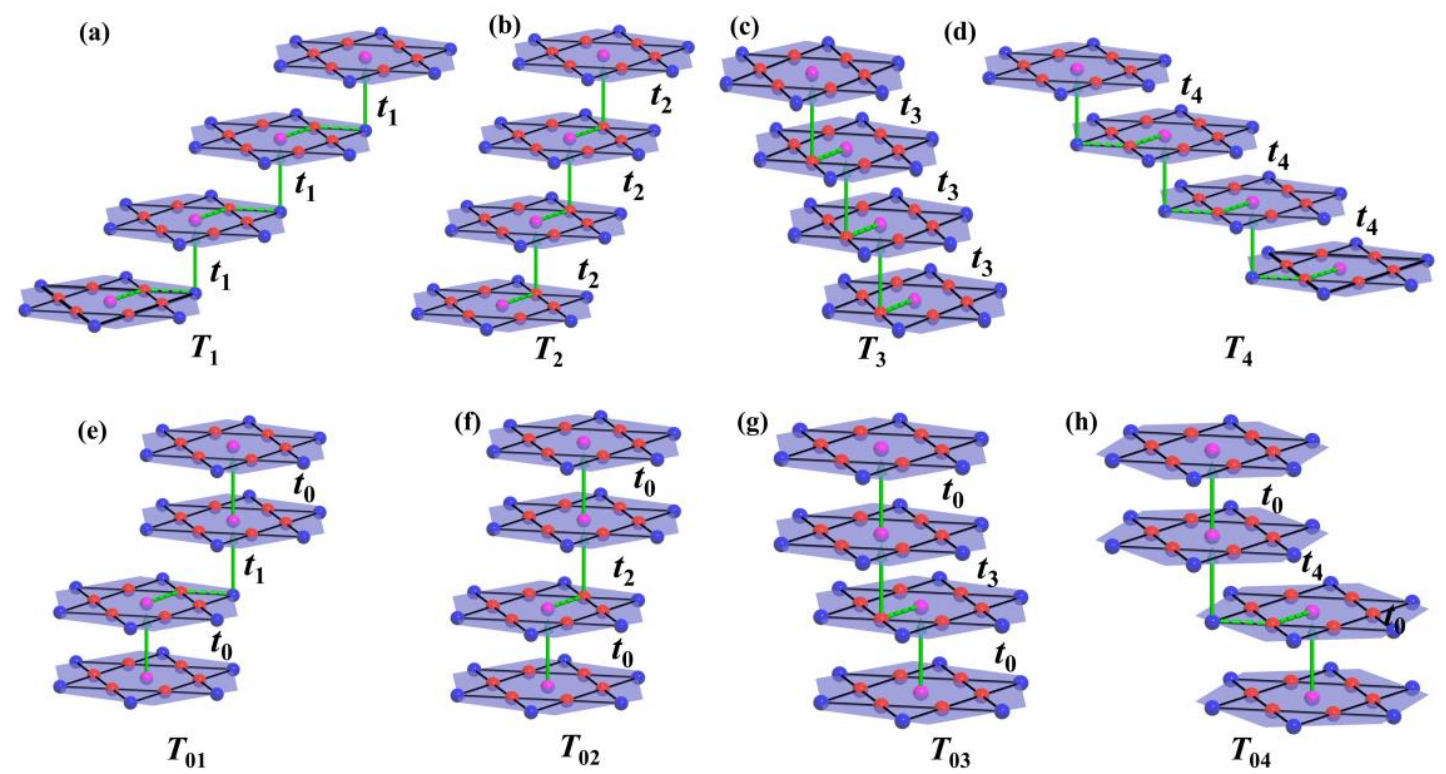

Figure 3 Schematics of the out-of-plane stacking order (the $\mathrm{S}$ atoms are not shown) of the SD clusters in the CCDW phase of $1 T-\mathrm{NbS}_{2}$ : (a)-(d) are four kinds of stacking patterns of $T_{1}-T_{4}$ with corresponding interlayer interface $t_{\mathrm{i}}\left(t_{1}-t_{4}\right)$ between two adjacent layers, (e)-(h) are four kinds of bilayer stacking patterns of $T_{01}-T_{04}$ with corresponding interlayer interface $t_{\mathrm{i}}\left(t_{1}-t_{4}\right)$ between two adjacent on-top stacked bilayers with $t_{0}$ interface.

Based on DFT, we performed all of the calculations by using Vienna ab initio simulation package (VASP) [43,44] within the generalized gradient approximation (GGA) [45] approach and projector-augmented wave (PAW) [46] potentials. The kinetic-energy cutoff of $520 \mathrm{eV}$ was used, and Brillouin zone (BZ) was segmented by Gamma centered grid of $0.2 \AA^{-1}$ for all structures.[47] Considering the weak interlayer interactions in the CCDW phase, vdW dispersion correction of DFT-D3 [48] was employed to perform geometrical optimization so that accurate lattice information and total energy can be obtained. Among the members of the $M X_{2}(M=\mathrm{Nb}, \mathrm{Ta} ; X=\mathrm{S}$, $\mathrm{Se})$ family, the $\mathrm{NbS}_{2}$ is composed of the lighter atoms and therefore is the most correlated one. To deal with the electronic correlation, we add the Coulomb repulsion $U$ on the $4 d$ orbitals of the $\mathrm{Nb}$ ranging from 0 to $3.5 \mathrm{eV}$ that are typical values for $4 d$ orbitals.

\section{RESULTS AND DISCUSSIONS}

Firstly, the normal on-top vertically stacking order $T_{0}$ of the CCDW phase [Figure 2(b)] serves as the starting point of our study. The optimized lattice parameters are $a=12.129 \AA$ and $c=$ $5.926 \AA$ at the DFT-D3 level, which are consistent with previous results.[30] In agreement with our previous results,[30] without electronic correlations the CCDW phase always converges to a 
nonmagnetic (NM) metallic state independent on the preset magnetic configuration. When we add the Coulomb interaction, the magnetic ground state is an interlayer AFM state that simultaneously opens the band gap [Figure S1 in the Supplemental Material (SM)].[49] However, as shown in Table 1, the calculated results indicate that the stacking order plays an essential impact on the layer spacing and stability. The total energy of the system depends remarkably on the stacking order and varies up to $\sim 60 \mathrm{meV} / \mathrm{SD}$. Compared with the $T_{0}$ stacking order, the layer spacing shrinks a bit and results in significant changes of the vdW energy, where the vdW energy $\Delta E_{\mathrm{vdw}}$ is positively proportional to the change of the layer spacing $\Delta c$. The vdW energy decreases as the layer spacing shrinks, demonstrating the crucial role of the vdW interactions is to anchor the layers.[50]

Table 1 Equilibrium layer spacing $(c)$ and energy landscape of the different stacking configurations for the NM states calculated within GGA. The changes of the layer spacing $(\Delta c)$, total energy $\left(\Delta E_{\mathrm{tot}}\right)$, and $\mathrm{vdW}$ energy $\left(\Delta E_{\mathrm{vdw}}\right)$ are calculated with respect to the $T_{0}$ stacking.

\begin{tabular}{ccccc}
\hline \hline Stacking order & $c(\AA)$ & $\Delta c(\%)$ & $\Delta E_{\mathrm{tot}}(\mathrm{meV} / \mathrm{SD})$ & $\Delta E_{\mathrm{vdw}}(\mathrm{meV} / \mathrm{SD})$ \\
\hline$T_{0}$ & 5.926 & 0 & 0 & 0 \\
$T_{1}$ & 5.887 & -0.658 & -24.82 & -75.77 \\
$T_{2}$ & 5.910 & -0.270 & 6.45 & -29.72 \\
$T_{3}$ & 5.909 & -0.287 & 12.11 & -31.28 \\
$T_{4}$ & 5.923 & -0.439 & 27.59 & -46.79 \\
$T_{01}$ & 5.908 & -0.304 & -36.29 & -36.54 \\
$T_{02}$ & 5.920 & -0.101 & -4.57 & -11.58 \\
$T_{03}$ & 5.920 & -0.101 & 0.21 & -10.96 \\
$T_{04}$ & 5.923 & -0.051 & -5.51 & -4.87 \\
\hline \hline
\end{tabular}

Furthermore, two stacking configurations of $T_{1}$ and $T_{01}$ exhibit remarkable stability from an energetic viewpoint. $T_{1}$ stacking order is the most favorable stacking arrangement for the vdW energy. On the contrary, although the benefit of $\mathrm{vdW}$ energy is not significant, $T_{01}$ stacking is preferred by total energy. Both $T_{1}$ and $T_{01}$ stacking orders are featured by the highly stable $t_{1}$ interface over the other interfaces, which are closely related to the favorable van der Waals interaction and interlayer S-S bonding.[21] In fact, the interlayer S-atoms configurations in different stacking systems are distinct from each other upon the specific stacking order. Shorter or longer S-S distances can be observed in the layer spacing. Such a steric effect of the S-atoms plays a significant impact on the energy of the stacking system. A shorter S-S distance results in an 
interlayer bonding with the $3 p_{z}$ orbitals, which is favorable for energy, but such a bonding interaction is disallowed for a longer S-S distance. As exemplified by $T_{1}$ and $T_{4}$ stacking orders, we can clearly identify the different interlayer distances between the nearest neighbor S-atoms coordinated with the central Nb-atoms of the SD cluster [Figures 4(a) and (b)]. As shown in the corresponding charge density maps [Figures 4(c) and (d)], bonding behavior can be clearly observed for the $T_{1}$ stacking with an interlayer S-S distance of $\sim 3.29 \AA$, whereas no such trace for the $T_{4}$ stacking with the interlayer S-S distance of $\sim 4.71 \AA$.

(a)

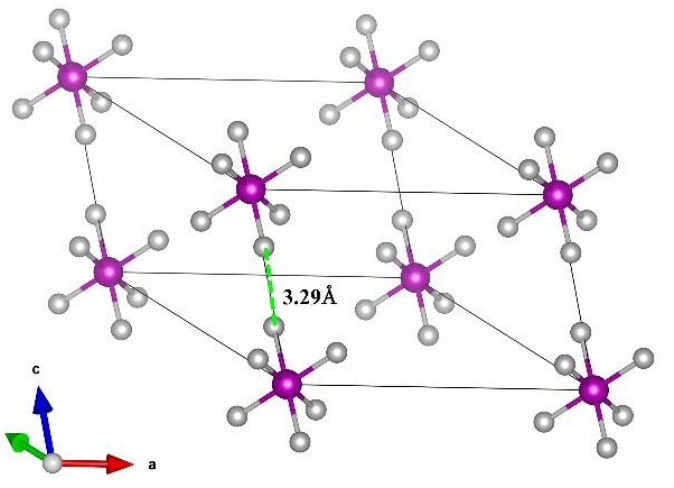

(c)

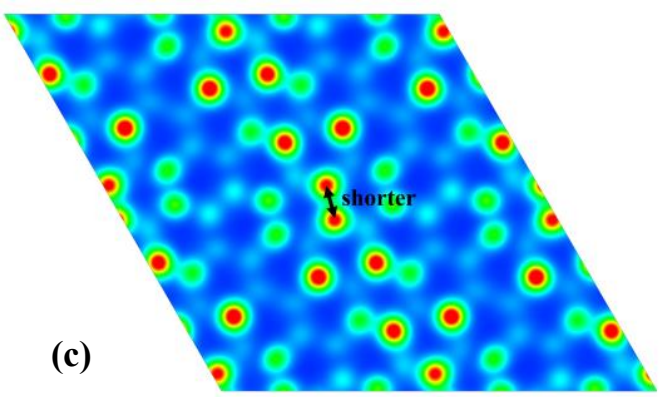

(b)

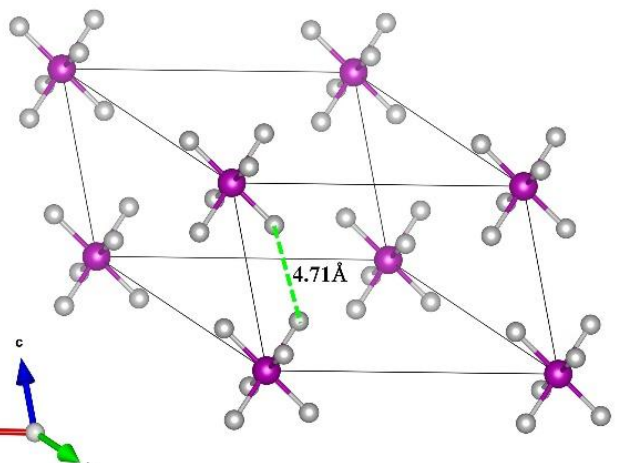

(d)

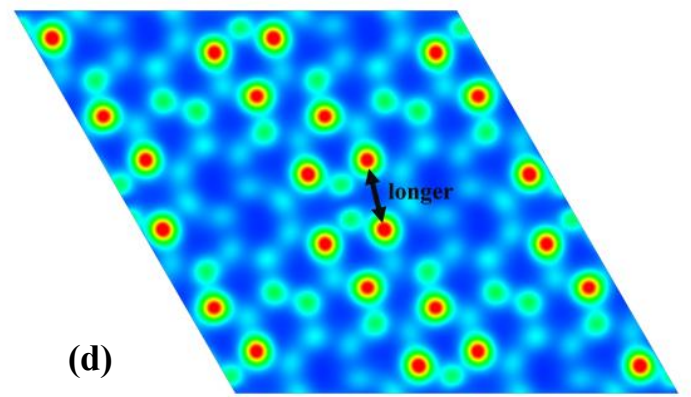

Figure 4 (a) and (b) are side views of the $T_{1}$ and $T_{4}$ stacking order (only the central $\mathrm{Nb}$-atoms of the SD cluster and the coordinated S-atoms are shown). (c) and (d) are charge density maps corresponding to the $T_{1}$ and $T_{4}$ stacking order, respectively, obtained by integrating electronic states near the Fermi level. The slices are located at the center of $t_{1}$ and $t_{4}$ interfaces and parallel to the $a b$ plane.

In addition, the electronic structures show obvious dependence on the stacking arrangements of the CCDW phase (see Figure 5 and Figure $\mathbf{S 2}$ in the SM).[49] In particular, the relative position of the SD clusters in adjacent $a b$-planes has a significant influence on the band dispersions. All the $T_{\mathrm{i}}$ stacking patterns show metallic behavior, whereas the $T_{0 \mathrm{i}}$ stacking arrangements show striking semiconductor-metal transitions. As shown in Figure 5(a), the $T_{1}$ stacking is metallic with a half-filled band characterized by the $4 d_{3 z^{2}-r^{2}}$ orbital contribution from the central Nb-atom of the SD clusters. Due to the presence of the $4 d_{3 z^{2}-r^{2}}$ bands at the 
Fermi level, the electronic properties strongly depend on the stacking along the $z$-axis. Attributed to the interlayer S-S bonding interactions [Figure 4 (a)], the $T_{1}$ stacking order shows a significant in-plane hopping [17] and stronger three-dimensional metallicity, where the bands cross the Fermi level not only along $\Gamma-A$ but also along $L-A$ and $L-H$. By contrast, the special orbital order intertwined with the CDW in the $T_{0}$ stacking only permits significant out-of-plane charge hopping, corresponding to the in-plane insulating characteristics along $\Gamma-M-K$ and out-of-plane one-dimensional metallic band dispersion along $\Gamma-A$.[30] Furthermore, as shown in Figure 5(b), an inherent band gap of $45.9 \mathrm{meV}$ is opened up for the $T_{01}$ stacking within GGA approach, implying a band insulating behavior instead of Mott insulator. Each cell is constructed from two SD clusters, and a pair of degenerate bands is almost completely isolated in the uppermost valence band. The contributions of the $4 d_{3 z^{2}-r^{2}}$ characteristics from the two central Nb- $0_{1}$ and $\mathrm{Nb}-\mathrm{O}_{2}$ atoms of the two SD are almost the same, indicating a degeneracy of them. A cell with an even number of electrons can yield filled valence bands forming an insulator or semiconductor.[51] Obviously, two orphan electrons from $\mathrm{Nb}-\mathrm{O}_{1}$ and $\mathrm{Nb}-\mathrm{O}_{2}$ pair with each other and fill the isolated orbit, accompanied by an opening of the band gap and a lowering of the total energy. Therefore, the stacking arrangements enable one to manipulate the band dispersion and gap structure of the CCDW phase $1 T-\mathrm{NbS}_{2}$. Similar to $1 T-\mathrm{TaS}_{2}$, the stacking order yields a new device concept, which utilizes metastable stacking orders to control the electronic structure of nanostructures and causes a semiconductor-metal transition.[17]
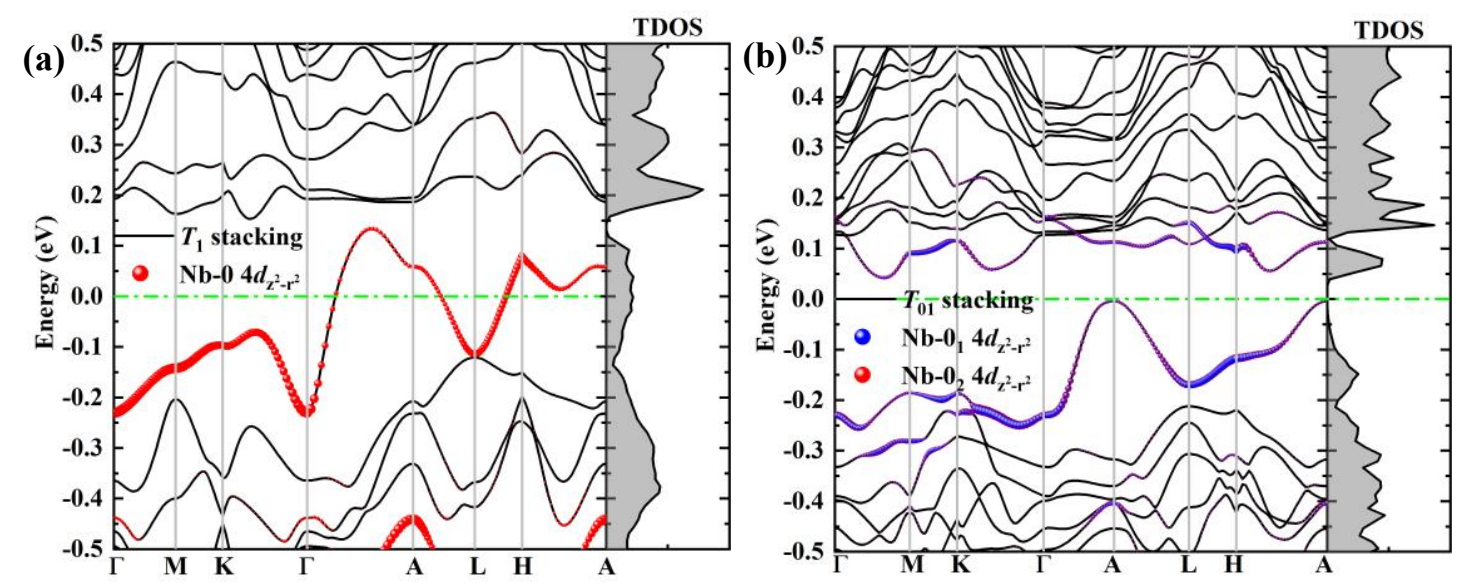

Figure 5 Band structures and corresponding density of states of the (a) $T_{1}$ and (b) $T_{01}$ stacking within GGA approach. The red balls in (a) denote the orbital contributions with $4 d_{3 z^{2}-r^{2}}$ character of the central $\mathrm{Nb}$ atom of the $\mathrm{SD}$ cluster $(\mathrm{Nb}-0)$. The blue/red balls in (b) denote the orbital contributions with $4 d_{3 z^{2}-r^{2}}$ character of $\mathrm{Nb}-0_{1} / \mathrm{Nb}-\mathrm{O}_{2}$ (central $\mathrm{Nb}$ atom of the $\mathrm{SD}$ in the 
first/second layer).

Attributed to the stacking effects, a band insulator is identified by interlayer dimerization in $T_{01}$ stacking of the CCDW phase $1 T-\mathrm{NbS}_{2}$, which is in contrast to the Mott-Hubbard mechanism proposed in previous studies on the origin of the insulating nature in the CCDW phase of $1 T-\mathrm{TaS}_{2}$ and monolayer $1 T-\mathrm{NbS}_{2} \cdot[23,31]$ However, recent studies on $1 T-\mathrm{TaS}_{2}$ have also implied that this dimerization cannot be simply depicted by a genuine Peierls mechanism if the continuity of order parameter has been taken into account, which is strongly related to the interlayer hopping.[16-18,21,22,24] It is worth noting that the $T_{01}$ stacking consists of two interfaces: the $t_{0}$ interface inside each on-top stacked paired bilayer and the $t_{1}$ interface between the adjacent bilayers. Once a stacking order is established, the interlayer hopping integral between adjacent layers will be changed dramatically, which has an essential impact on the electronic structure. The interlayer hopping in the $t_{0}$ interface is the same in the $T_{0}$ stacking, while interlayer hopping is much greater in the $t_{0}$ interface than the $t_{1}$ interface in the $T_{01}$ stacking.[18] Moreover, there exists a continuous crossover region between the band insulating and Mott insulating phase in the bilayer Hubbard system, and the stronger interlayer hopping is beneficial to the band insulator.[52,53] Similar to the previous findings for $1 T-\mathrm{TaS}_{2}$, the strong electron-electron correlation does not play a major role in the insulating nature of the $T_{01}$ stacking, whereas the on-top stacked bilayer and the interlayer hopping should be the most important factors.[16,21]

Despite the band insulating behavior can be realized by paired SD layers without considering the electron-electron correlation,[16,17,21] the strong correlations and Mott physics still exist in the limit of monolayers.[31] Furthermore, the orphan electron on each SD cluster provides $S=1 / 2$ spin moment in the triangle lattice, which offers an opportunity to study the Coulomb correlation and quantum state in the CCDW phase of bulk $1 T-\mathrm{NbS}_{2}$. By considering Coulomb correlations at the level of $U=2.95 \mathrm{eV},[30]$ we calculate the energy differences between different stacking configurations to explore the effects of the electron-electron correlation on the relative stability and the electronic structures. Especially, by doubling the cell along the layer stacking direction, two magnetic ordering states of AFM $\downarrow$ and $\mathrm{FM} \uparrow \uparrow$ are preset for $T_{\mathrm{i}}$ stacking, whereas four magnetic states of AFM $\downarrow \uparrow \downarrow$, AFM $\uparrow \downarrow \uparrow, \mathrm{AFM} \uparrow \uparrow \downarrow \downarrow$ and $\mathrm{FM} \uparrow \uparrow \uparrow \uparrow$ are considered for $T_{0 \mathrm{i}}$ stacking. As shown in Figure 6, the magnetic ordering states exhibit lower energy relative to the NM state for all stacking patterns, which implies possible magnetic states at low temperature. The 
unpaired single-layer $T_{\mathrm{i}}$ stacking patterns, especially the $T_{1}$ and $T_{4}$ stacking arrangements show a stronger response to the magnetic ordering and Coulomb correlation. By contrast, the energy gains of the $T_{0 \mathrm{i}}$ stacking are not so striking, that the energy of the AFM state and the NM state differs only a few meV/SD. Moreover, the distinct stacking arrangements and interfaces show different magnetic configurations. For instance, the $T_{4}$ stacking tends to form $\mathrm{FM} \uparrow \uparrow$ state rather than AFM $\uparrow \downarrow$ state, and $T_{04}$ stacking tends to form AFM $\downarrow \downarrow \downarrow \uparrow$ state rather than AFM $\uparrow \uparrow \uparrow \downarrow$ state, implying that the $t_{4}$ interface is prone to form FM $\uparrow \uparrow$ configuration. In accord with the $T_{0}$ stacking, the $t_{0}$ interface tends to form AFM paired bilayers, all the $T_{0 \mathrm{i}}$ stacking orders are featured with $\mathrm{AFM} \uparrow \downarrow$ configuration in the $t_{0}$ interface. Therefore, a variation of the stacking orders by a lateral sliding could change the direct hopping strength between interlayer S orbitals, which further alters the interlayer magnetic interactions between FM and AFM ones. The stacking order provides an effective way to tailor the interlayer magnetic interactions, which opens a paradigm for seeking metamagnetic 2D materials from AFM to FM state in real applications.[14,15]

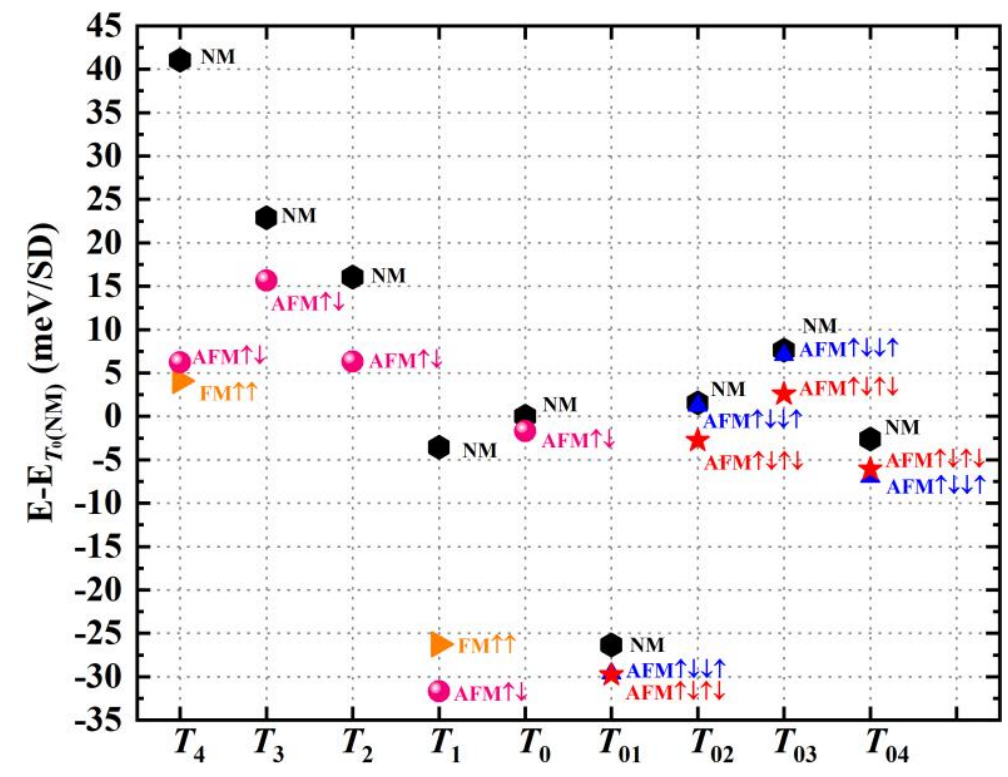

Figure 6 Energy landscape of different stacking order with respect to $T_{0}$ stacking of the NM state. All magnetic ordering states are calculated by GGA $+U$ within the Coulomb correlation of $U=$ $2.95 \mathrm{eV}$. The upward and downward arrows represent the directions of the spin magnetic moments in different layers. We should note that not all our preset magnetic configurations are presented here, because the unstable magnetic states have converged to NM state or other states.

The $T_{1}$ and $T_{01}$ stacking configurations are still far more stable than the other stacking orders (by $\sim 30 \mathrm{meV} / \mathrm{SD}$ ) (Figure 6). Surprisingly, the $T_{1}$ stacking obtains a huge energy gain after entering the magnetic ordering states, so that the total energy is lower than that of the $T_{01}$ stacking 
by about $1.9 \mathrm{meV} / \mathrm{SD}$. In addition, in contrast to the metallic band structure of the NM state, a tiny insulating gap of $12.9 \mathrm{meV}$ is opened up for the AFM state (Figure 7), which signifies the Mott-Slater insulating nature of the $T_{1}$ stacking (will be discussed in detail later). The electron-electron correlation and magnetic interactions play a minor impact on the $T_{01}$ stacking, the energy differences between the NM and AFM states are within only $3 \mathrm{meV} / \mathrm{SD}$. The two AFM states (AFM $\uparrow \downarrow \downarrow \uparrow$ and $\mathrm{AFM} \uparrow \downarrow \uparrow \downarrow$ ) of the $T_{01}$ stacking order almost have identical energies, indicating that the $T_{01}$ stacking is not sensitive to the interlayer magnetic configurations, and the typical characteristics of the band insulator are robust. This phenomenon is consistent with the recent experimental confirmation that the paired bilayer $T_{01}$ stacking is the dominant building block of $1 T-\mathrm{TaS}_{2}$ at low temperatures.[18,24] As stated above, the primary origin of the insulating properties in $T_{01}$ stacking is the dimerization and interlayer hopping, while strong electron-electron correlation does not play a key role. In other words, $T_{01}$ stacking is insensitive to Coulomb repulsion $U$, in contrast to the case of the $T_{1}$ stacking.

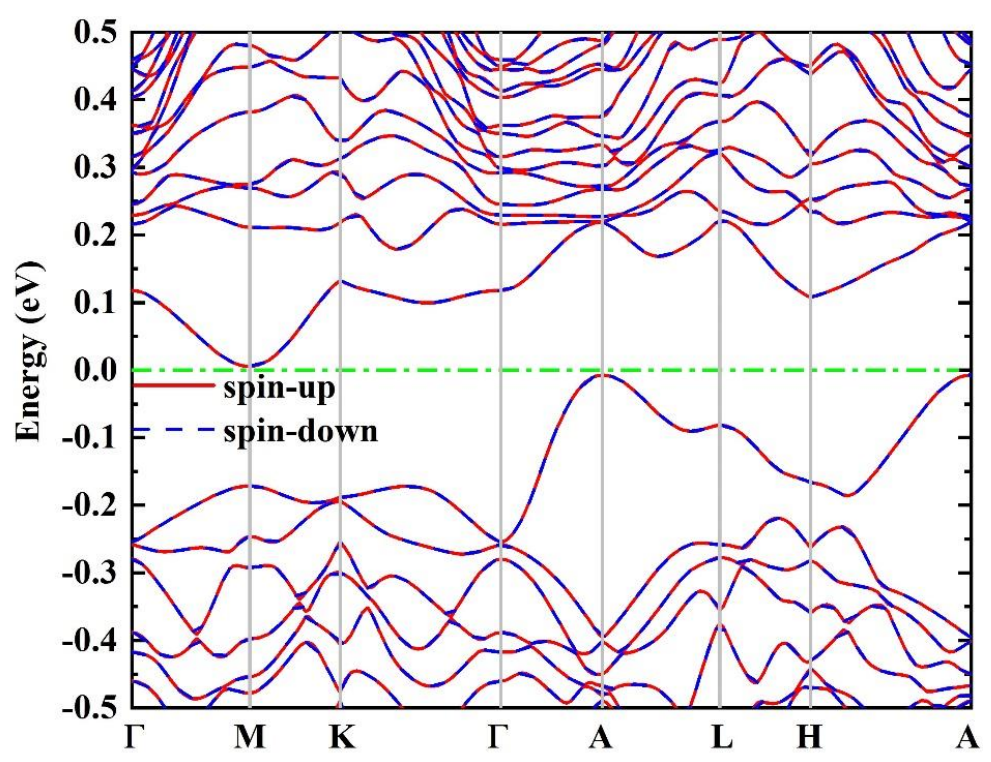

Figure 7 Band structure of the interlayer AFM state for $T_{1}$ stacking calculated by GGA $+U(U=$ $2.95 \mathrm{eV})$.

Compared with the $T_{01}$ stacking order, the distinct insulating mechanisms leads to the compensation of the total energy for $T_{1}$ stacking under the action of $U$, which eventually results in a lower-energy stacking order. Notable is that the total energy difference between the $T_{1}$ and $T_{01}$ stacking configurations is only $\sim 2 \mathrm{meV} / \mathrm{SD}$. The CCDW phase of bulk $1 T-\mathrm{NbS}_{2}$ provides a platform to reveal the underlying role of $U$ in the ground state with different stacking orders. 
Considering that the $\mathrm{Nb}$ element has a $4 d$ shell with moderate electron-electron correlation, we choose the $U$ value ranging from $0 \mathrm{eV}$ to $3.5 \mathrm{eV}$. As shown in Figure 8, the $T_{01}$ stacking order converges to NM insulating states when the $U$ values are lower than $2.5 \mathrm{eV}$, then the $\mathrm{NM}$ insulating state transforms to an AFM insulating state when the $U$ value reaches up to $2.5 \mathrm{eV}$. Dynamical mean-field theory studies on the bilayer Hubbard model have identified a crossover from band insulator to Mott insulator under the effect of increasing $U$ values.[52,53] On the contrary, the $T_{1}$ stacking order shows lower-energy NM metallic states when the $U$ values are lower than $1.5 \mathrm{eV}$, and the energy differences between the $T_{1}$ and $T_{01}$ stacking orders gradually increase with the increasing $U$ value. Once the $T_{1}$ stacking order transforms from the NM metallic to AFM metallic states at $U=1.75 \mathrm{eV}$, the evolution trend of the energy differences shows an upturn, which gradually decreases with the increasing $U$ values. When the $U$ value reaches up to $2.5 \mathrm{eV}$, the $T_{1}$ stacking order transforms from the AFM metallic state to an AFM insulating state. Therefore, both Coulomb repulsion and magnetic order are necessary to open the gap in the $T_{1}$ stacking order. As shown in other compounds, this is a signature of a Slater-Mott insulator.[54-56] We can claim that along with the increasing $U$ values, the $T_{1}$ stacking order exhibits an interesting Slater-Mott metal-insulator transition from NM metal to AFM metal and finally to AFM insulator. Along with the $U$ value further increasing, the energy differences between the $T_{1}$ and $T_{01}$ stacking orders gradually decrease. As a result, the ground state transforms from the band insulator to the Slater-Mott insulator. Previous calculations suggest that the band insulator may be so fragile that electronic doping or applying strain can easily destroy the relative stability of the two stacking configurations.[21] We speculate that the CCDW phase of bulk $1 T-\mathrm{NbS}_{2}$ locating at the crossover region of Slater-Mott insulator and band insulator, remaining to be uncovered experimentally. More precisely, both Coulomb repulsion and magnetism are needed to open the bandgap making these states a Slater-Mott insulator. 


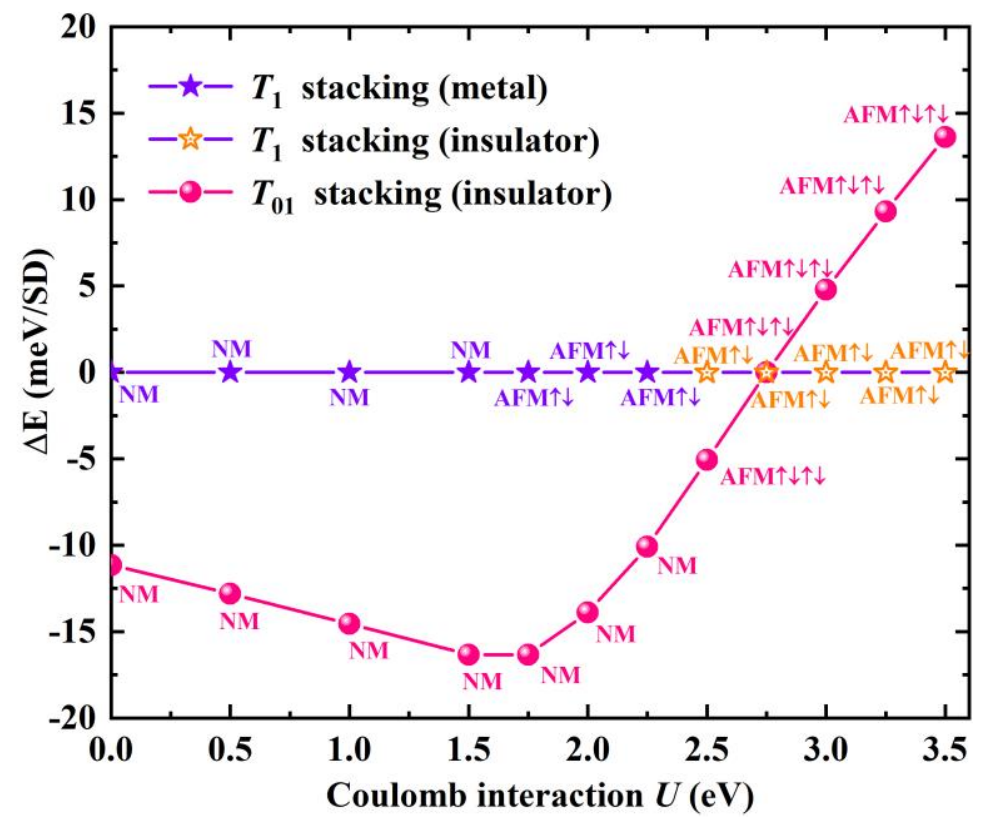

Figure 8 The evolutions of the energy differences and metal-insulator transitions between $T_{1}$ and $T_{01}$ stacking along with the increasing Coulomb correlation parameters $U$.

The bilayer-stacked bulk phase of $1 T-\mathrm{NbS}_{2}$ and $1 T-\mathrm{TaS}_{2}$ may satisfy the criteria of a simple band insulator, but ARPES and X-ray diffraction data have suggested certain randomness of the out-of-plane stacking order, and STEM topographic images show the broken dimerization by unpaired SD clusters at the surfaces of the CCDW phase $1 T-\mathrm{TaS}_{2} \cdot[18,24]$ These experiments demonstrate the possible existence of an unpaired stacking SD cluster accompanied with a small change of stacking order, which will yield band insulator to Mott insulator transitions and metal-insulator transitions. The small energy differences between the $T_{1}$ and $T_{01}$ stacking order jointed with the possible stacking faults and stacking disorder in real materials will give rise to complex phase diagrams for the CCDW phase of $1 T-\mathrm{NbS}_{2}$. Additionally, the electron-electron correlation cannot be absolutely precluded, Mott physics might still play an important role in the electronic structure of the CCDW phase.[16] We note that first-principles calculations within traditional DFT, DFT $+U$ and the hybrid functional have failed to reproduce the out-of-plane insulating behavior of the bulk $1 T-\mathrm{TaS}_{2}$ for the $T_{0}$ stacking order with on-top stacked SD clusters.[16,17,21,25,57] Recently, a Mott insulating band structure with the interlayer AFM order has been successfully achieved by applying the $U$ potential onto a generalized basis.[58] However, any magnetic ordering has never been observed in $1 T-\mathrm{TaS}_{2}$ so far.[39-42] In contrast, the calculated electronic structures show a band insulating behavior without considering the magnetic 
configurations in the CCDW phase of $1 T-\mathrm{NbS}_{2}$, which transforms to Slater-Mott insulating state by considering the magnetic ordering states and Coulomb correction with DFT $+U$. Furthermore, the energy differences of the magnetic ordering states between the most stable $T_{1}$ and $T_{01}$ stacking order are very small, which implies a possible magnetic disordering associated with the triangular lattice. These stacking-driven metal-insulator transitions are similar to thickness-driven metal-insulator transitions in transition-metal oxides, where in both cases the structural effects are responsible for a bandwidth reduction and consequently for a thickness-controlled metal-insulator transition towards more correlated electronic phases.[54-56] The presence of different electronic and magnetic phases paves the way to manipulate the competition between these phases by doping or applied external pressure. The concomitant insulating nature, localized orphan spin, and possible magnetic disordering on the triangular lattice of CCDW phase $1 T-\mathrm{NbS}_{2}$ will induce exotic quantum states, perhaps the elusive quantum spin liquid, needing to be further clarified both theoretically and experimentally.

\section{CONCLUSIONS}

In summary, we explored the influences of stacking order and electron correlation on the electronic properties of the CCDW phase $1 T-\mathrm{NbS}_{2}$ by first-principles calculations. The electronic structures show a significant dependence on the stacking order. Without considering the electron-electron correlation, two energetic favorable stacking orders of $T_{1}$ and $T_{01}$ show distinct electronic structures, one is metal and the other one is band insulator. These two energetic favorable stacking orders are still robust while considering the electron-electron correlation. Particularly, the ground state of the system is strongly related to the strength of the electron-electron correlation. Along with the increasing Coulomb interactions, the $T_{1}$ stacking order undergoes a Slater-Mott metal-insulator transition, whereas the $T_{01}$ stacking order transforms from band insulator to AFM insulator, indicating the indispensability of electron correlation. Our work highlights the critical role of interlayer stacking order and reveals an important role of the Coulomb correlation in the electronic properties of the CCDW phase $1 T-\mathrm{NbS}_{2}$. Furthermore, we propose stacking-controllable metal-insulator transitions, metamagnetic transitions, and exotic quantum phase perhaps existing in the promising $1 T-\mathrm{NbS}_{2}$, which will draw intensive attention.

\section{ACKNOWLEDGMENTS}

We would like to thank the fruitful discussion with Dr. Sung-Hoon Lee from Kyung Hee 
University. The work was sponsored by the National Natural Science Foundation of China (No. 11864008) and Guangxi Natural Science Foundation (No. 2018AD19200 and 2019GXNSFGA245006). C. A. is supported by the Foundation for Polish Science through the International Research Agendas program co-financed by the European Union within the Smart Growth Operational Programme.

\section{REFERENCES}

1. Fiori, Gianluca, et al. "Electronics based on two-dimensional materials." Nature Nanotechnology 9 (2014): 768.

2. Geim, Andre K., and Irina V. Grigorieva. "Van der Waals heterostructures." Nature 499 (2013): 419.

3. Novoselov, K. S., et al. "2D materials and van der Waals heterostructures." Science $\mathbf{3 5 3}$ (2016): aac9439.

4. Carr, Stephen, et al. "Twistronics: Manipulating the electronic properties of two-dimensional layered structures through their twist angle." Physical Review B 95 (2017): 075420.

5. Ribeiro-Palau, Rebeca, et al. "Twistable electronics with dynamically rotatable heterostructures." Science 361 (2018): 690.

6. Cao, Yuan, et al. "Unconventional superconductivity in magic-angle graphene superlattices." Nature 556 (2018): 43.

7. Yankowitz, Matthew, et al. "Tuning superconductivity in twisted bilayer graphene." Science 363 (2019): 1059.

8. Lu, Xiaobo, et al. "Superconductors, orbital magnets and correlated states in magic-angle bilayer graphene." Nature 574 (2019): 653.

9. Cao, Yuan, et al. "Correlated insulator behaviour at half-filling in magic-angle graphene superlattices." Nature 556 (2018): 80.

10. Sharpe, Aaron L., et al. "Emergent ferromagnetism near three-quarters filling in twisted bilayer graphene." Science 365 (2019): 605.

11. Serlin, M., et al. "Intrinsic quantized anomalous Hall effect in a moiré heterostructure." Science 367 (2020): 900.

12. Li, Lei, and Menghao Wu. "Binary compound bilayer and multilayer with vertical polarizations: two-dimensional ferroelectrics, multiferroics, and nanogenerators." ACS nano 11 (2017): 6382.

13. Yasuda, Kenji, et al. "Stacking-engineered ferroelectricity in bilayer boron nitride." Science 372 (2021): 1458.

14. Jiang, Peiheng, et al. "Stacking tunable interlayer magnetism in bilayer $\mathrm{CrI}_{3} . "$ Physical Review B 99 (2019): 144401.

15. Chen, Weijong, et al. "Direct observation of van der Waals stacking-dependent interlayer magnetism." Science 366 (2019): 983. 
16. Ritschel, T., H. Berger, and J. Geck. "Stacking-driven gap formation in layered $1 T-\mathrm{TaS}_{2} . "$ Physical Review B 98 (2018): 195134.

17. Ritschel, T., et al. "Orbital textures and charge density waves in transition metal dichalcogenides." Nature Physics 11 (2015): 328.

18. Butler, C. J., et al. "Mottness versus unit-cell doubling as the driver of the insulating state in 1T-TaS $2 . "$ Nature Communications 11 (2020): 2477.

19. Ma, Liguo, et al. "A metallic mosaic phase and the origin of Mott-insulating state in 1T-TaS $2 . "$ Nature Communications 7 (2016): 10956.

20. Fazekas, P., and E. Tosatti. "Charge carrier localization in pure and doped $1 T$-TaS $\mathrm{T}_{2}$." Physica B+C 99 (1980): 183.

21. Lee, Sung-Hoon, Jung Suk Goh, and Doohee Cho. "Origin of the Insulating Phase and First-Order Metal-Insulator Transition in 1T-TaS 2." Physical Review Letters 122 (2019): 106404.

22. Martino, Edoardo, et al. "Preferential out-of-plane conduction and quasi-one-dimensional electronic states in layered 1T-TaS $2 . "$ npj 2D Materials and Applications 4 (2020): 7.

23. Yu, Xiang-Long, et al. "Electronic correlation effects and orbital density wave in the layered compound 1T-TaS $2 . "$ Physical Review B 96 (2017): 125138.

24. Wang, Y. D., et al. "Band insulator to Mott insulator transition in $1 T$ - $\mathrm{TaS}_{2}$." Nature Communications 11 (2020): 4215.

25. Darancet, Pierre, Andrew J. Millis, and Chris A. Marianetti. "Three-dimensional metallic and two-dimensional insulating behavior in octahedral tantalum dichalcogenides." Physical Review B 90 (2014): 045134.

26. von Witte, Gevin, et al. "Surface structure and stacking of the commensurate $(\sqrt{13} \times \sqrt{13})$ R13.9॰ charge density wave phase of $1 T-\mathrm{TaS}_{2}$ (0001)." Physical Review B 100 (2019): 155407.

27. Hovden, Robert, et al. "Atomic lattice disorder in charge-density-wave phases of exfoliated dichalcogenides (1T-TaS 2$)$." Proceedings of the National Academy of Sciences 113 (2016): 11420 .

28. Wu, Zongxiu, et al. "Effect of Stacking Order on the Electronic State of $1 T-\mathrm{TaS}_{2}$." arXiv preprint arXiv:2105.08663 (2021).

29. Cho, Doohee, et al. "Nanoscale manipulation of the Mott insulating state coupled to charge order in 1T-TaS $2 . "$ Nature Communications 7 (2016): 10453.

30. Wei Wang, Bosen Wang, Zhibin Gao, Gang Tang, Wen Lei, Xiaojun Zheng, Huan Li, Xing Ming, and Carmine Autieri. "Charge density wave instability and pressure-induced superconductivity in bulk 1T-NbS2." Physical Review B 102 (2020): 155115.

31. Tresca, Cesare, and Matteo Calandra. "Charge density wave and spin 1/2 insulating state in single layer $1 T-\mathrm{NbS}_{2} . "$ 2D Materials 6 (2019): 035041.

32. Wang, Wei, et al. "Electronic structure and phase transition engineering in $\mathrm{NbS}_{2}$ : Crucial role 
of van der Waals interactions." Chinese Physics B 29 (2020): 056201.

33. El Youbi, Zakariae, et al. "Fermiology and electron-phonon coupling in the $2 H$ and $3 R$ polytypes of $\mathrm{NbS}_{2}$." Physical Review B 103 (2021): 155105.

34. Leroux, Maxime, et al. "Traces of charge density waves in $\mathrm{NbS}_{2}$." Physical Review B 97 (2018): 195140.

35. Guillamón, I., et al. "Superconducting density of states and vortex cores of $2 \mathrm{H}-\mathrm{NbS}_{2} . "$ Physical Review Letters 101 (2008): 166407.

36. Martino, Edoardo, et al. "Unidirectional Kondo scattering in layered $\mathrm{NbS}_{2}$." arXiv preprint arXiv:2104.09147 (2021).

37. Law, Kam Tuen, and Patrick A. Lee. "1T-TaS 2 as a quantum spin liquid." Proceedings of the National Academy of Sciences 114 (2017): 6996.

38. He, Wen-Yu, et al. "Spinon Fermi Surface in a Cluster Mott Insulator Model on a Triangular Lattice and Possible Application to 1T-TaS 2 ." Physical Review Letters 121 (2018): 046401.

39. Klanjšek, Martin, et al. "A high-temperature quantum spin liquid with polaron spins." Nature Physics 13 (2017): 1130.

40. Kratochvilova, Marie, et al. "The low-temperature highly correlated quantum phase in the charge-density-wave 1T-TaS 2 compound." npj Quantum Materials 2 (2017): 42.

41. Ribak, Amit, et al. "Gapless excitations in the ground state of $1 T-\mathrm{TaS}_{2} . "$ Physical Review B 96 (2017): 195131.

42. Mañas-Valero, Samuel, et al. "Quantum phases and spin liquid properties of $1 T-\mathrm{TaS}_{2}$." npj Quantum Materials 6 (2021): 69.

43. Kresse, Georg, and Jürgen Hafner. "Ab initio molecular dynamics for liquid metals." Physical Review B 47 (1993): 558.

44. Kresse, Georg, and Jürgen Furthmüller. "Efficiency of ab-initio total energy calculations for metals and semiconductors using a plane-wave basis set." Computational Materials Science 6 (1996): 15.

45. Perdew, John P., Kieron Burke, and Matthias Ernzerhof. "Generalized gradient approximation made simple." Physical Review Letters 77 (1996): 3865.

46. Kresse, Georg, and Daniel Joubert. "From ultrasoft pseudopotentials to the projector augmented-wave method." Physical Review B 59 (1999): 1758.

47. Monkhorst, Hendrik J., and James D. Pack. "Special points for Brillouin-zone integrations." Physical Review B 13 (1976): 5188.

48. Grimme, Stefan. "Semiempirical GGA-type density functional constructed with a long-range dispersion correction." Journal of Computational Chemistry 27 (2006): 1787.

49. See Supplemental Material for the nonmagnetic and antiferromagnetic band structure of the $1 T-\mathrm{NbS}_{2} \mathrm{CCDW}$ phase within $T_{0}$ stacking configuration, and nonmagnetic state band structure of the $T_{2}, T_{3}, T_{4}, T_{02}, T_{03}$, and $T_{04}$ stacking configurations.

50. Marom, Noa, et al. "Stacking and registry effects in layered materials: the case of hexagonal 
boron nitride." Physical Review Letters 105 (2010): 046801.

51. Naito, Michio, Hironori Nishihara, and Shoji Tanaka. "Nuclear Magnetic Resonance and Nuclear Quadrupole Resonance Study of ${ }^{181} \mathrm{Ta}$ in the Commensurate Charge Density Wave State of $1 T-\mathrm{TaS}_{2}$." Journal of the Physical Society of Japan $\mathbf{5 5}$ (1986): 2410.

52. Fuhrmann, Andreas, David Heilmann, and Hartmut Monien. "From Mott insulator to band insulator: A dynamical mean-field theory study." Physical Review B 73 (2006): 245118.

53. Kancharla, S. S. and Okamoto, S. "Band insulator to Mott insulator transition in a bilayer Hubbard model." Physical Review B 75 (2007): 193103.

54. Watanabe, Hiroshi, Tomonori Shirakawa, and Seiji Yunoki. "Theoretical study of insulating mechanism in multiorbital Hubbard models with a large spin-orbit coupling: Slater versus Mott scenario in $\mathrm{Sr}_{2} \mathrm{IrO}_{4}$." Physical Review B 89 (2014): 165115.

55. Yang, Junyi, et al. "Strain-Modulated Slater-Mott Crossover of Pseudospin-Half Square-Lattice in $\left(\mathrm{SrIrO}_{3}\right)_{1} /\left(\mathrm{SrTiO}_{3}\right)_{1}$ Superlattices." Physical Review Letters 124 (2020): 177601.

56. Groenendijk, D. J., et al. "Spin-orbit semimetal $\mathrm{SrIrO}_{3}$ in the two-dimensional limit." Physical Review Letters 119 (2017): 256403.

57. Lazar, Petr, Jana Martincová, and Michal Otyepka. "Structure, dynamical stability, and electronic properties of phases in $\mathrm{TaS}_{2}$ from a high-level quantum mechanical calculation." Physical Review B 92 (2015): 224104.

58. Shin, Dongbin, et al. "Identification of the Mott Insulating Charge Density Wave State in 1T-TaS $2 . "$ Physical Review Letters 126 (2021): 196406. 